\title{
AN EXAMPLE OF A TWO-TERM ASYMPTOTICS FOR THE "COUNTING FUNCTION" OF A FRACTAL DRUM
}

\author{
JACQUELINE FLECKINGER-PELLÉ AND DMITRI G. VASSILIEV
}

\begin{abstract}
In this paper we study the spectrum of the Dirichlet Laplacian in a bounded domain $\Omega \subset \mathbb{R}^{n}$ with fractal boundary $\partial \Omega$. We construct an open set $\mathscr{Q}$ for which we can effectively compute the second term of the asymptotics of the "counting function" $N(\lambda, \mathscr{Q})$, the number of eigenvalues less than $\lambda$. In this example, contrary to the $M$. V. Berry conjecture, the second asymptotic term is proportional to a periodic function of $\ln \lambda$, not to a constant. We also establish some properties of the $\zeta$-function of this problem. We obtain asymptotic inequalities for more general domains and in particular for a connected open set $\mathscr{O}$ derived from $\mathscr{Q}$. Analogous periodic functions still appear in our inequalities. These results have been announced in [FV].
\end{abstract}

\section{INTRODUCTION}

Let $\Omega$ be a bounded open set in $\mathbb{R}^{n} \quad(n \geq 2)$, with "fractal" (very irregular, see below) boundary $\partial \Omega$. We consider the following eigenvalue problem-in its variational sense:

$$
\begin{gathered}
-\Delta u=\lambda u \quad \text { in } \Omega, \\
u=0 \quad \text { on } \partial \Omega .
\end{gathered}
$$

More precisely we say that $\lambda$ is an eigenvalue of (1.1) if there exists a nonzero $u$ in $H_{0}^{1}(\Omega)$ satisfying the equation (1.1.a) in its distributional sense. Here $H_{0}^{1}(\Omega)$ is the completion of $\mathscr{D}(\Omega)$ with respect to the Sobolev norm $\|\cdot\|_{H^{1}(\Omega)}$. Since $\Omega$ is bounded the spectrum of problem (1.1) consists of a countable sequence of positive eigenvalues $\lambda_{j}(\Omega)$ (each eigenvalue being repeated according to multiplicity):

$$
0<\lambda_{1}(\Omega)<\lambda_{2}(\Omega) \leq \cdots \leq \lambda_{j}(\Omega) \leq \cdots, \quad \lambda_{j}(\Omega) \rightarrow+\infty \quad \text { as } j \rightarrow+\infty .
$$

Let $\lambda$ be a positive given number. We denote by $N(\lambda, \Omega)$ the number of eigenvalues less than $\lambda$ :

$$
N(\lambda, \Omega)=\sharp\left\{\lambda_{j}(\Omega)<\lambda\right\} .
$$

$N(\lambda, \Omega)$ is usually referred to as the "counting function" of problem (1.1).

It is known that the following asymptotics holds:

$$
N(\lambda, \Omega) \sim W(\lambda, \Omega):=(2 \pi)^{-n} \omega_{n} \lambda^{n / 2}|\Omega|_{n} \quad \text { as } \lambda \rightarrow+\infty,
$$

Received by the editors December 14, 1990.

1980 Mathematics Subject Classification (1985 Revision). Primary 35P20. 
where $\omega_{n}$ is the volume of the unit ball in $\mathbb{R}^{n}$ and $|\cdot|_{n}$ denotes the $n$ dimensional Lebesgue measure in $\mathbb{R}^{n}$. Formula (1.3) was established in 1911 by $\mathrm{H}$. Weyl [W1, W2] for smooth boundaries and then extended to irregular boundaries in [BS, FM, Mt1, Mt2].

In the case of smooth boundaries and under some other assumptions (there are not too many periodic geodesics) one has [Iv1, Iv2, M11, M12, V1]:

$$
N(\lambda, \Omega)=W(\lambda, \Omega)-\kappa_{n}|\partial \Omega|_{n-1} \lambda^{(n-1) / 2}+o\left(\lambda^{(n-1) / 2}\right) \quad \text { as } \lambda \rightarrow+\infty,
$$

where $\kappa_{n}$ is a constant which depends only on $n$. When $\Omega$ is a Riemannian manifold and has symmetries (1.4) does not necessarily hold as shown in [DG, $\mathrm{S} 1, \mathrm{~S} 2]$.

Here we study the case of a fractal boundary $\partial \Omega$, i.e. a boundary with a noninteger dimension. Fractal boundaries have attracted much attention lately since they appear in many physical problems such as the detection of cracks, the catalysis on porous media etc.

In 1979 M. V. Berry studying the scattering of waves by "fractals"-and motivated by (1.4) - made the following conjecture for $\partial \Omega$ with fractal dimension $\delta \in(n-1, n)$ :

$$
N(\lambda, \Omega)=W(\lambda, \Omega)-\kappa_{n, \delta} \mu_{\delta}(\partial \Omega) \lambda^{\delta / 2}+o\left(\lambda^{\delta / 2}\right) \text { as } \lambda \rightarrow \infty ;
$$

in this conjecture $\delta$ is the Hausdorff dimension of the boundary $\partial \Omega$ and $\mu_{\delta}(\partial \Omega)$ is its $\delta$-dimensional Hausdorff measure.

It was then proved by [BC] that (1.5) cannot be true with $\delta$ being the Hausdorff dimension and the authors suggested to replace $\delta$ by $d_{i}$ - the interior Bouligand-Minkowski dimension of the boundary.

In [LF1, LF2], the following asymptotics was proved:

$$
N(\lambda, \Omega)=W(\lambda, \Omega)+O\left(\lambda^{d / 2}\right) \text { as } \lambda \rightarrow+\infty,
$$

where $d$ is the Bouligand-Minkowski dimension of the boundary which is assumed to have a finite upper Minkowski content. Extensions of (1.6) were established in [L1] and it was proved in [V2, L1, FV] that for our problem (1.1) (with Dirichlet boundary conditions) only $d_{i}$-as sketched in [BC]-can appear.

Here we construct an example which contradicts the "modified Berry conjecture" where $\delta$ is replaced by $d_{i}$; in our example the second term of the asymptotics of the counting function is not proportional to $\lambda^{d_{i} / 2}$; as for smooth Riemannian manifolds with strong symmetries, the second term oscillates and is of the form $-c p(\ln \lambda) \lambda^{d_{i} / 2}$ where $p$ is a positive periodic function as first suggested in [V2] and then announced in [FV].

Before giving our results, let us recall the definition of the interior BouligandMinkowski dimension of $\partial \Omega$. For a positive given number $\varepsilon$ we set

$$
\Gamma_{\varepsilon}^{i}=\{x \in \Omega: \operatorname{dist}(x, \partial \Omega)<\varepsilon\}
$$

where $\operatorname{dist}(\cdot, \cdot)$ denotes the Euclidean distance in $\mathbb{R}^{n}$. For a given $h>0$ we consider

$$
\mu^{*}(h, \partial \Omega)=\limsup _{\varepsilon \rightarrow+0} \varepsilon^{-(n-h)} \mid \Gamma_{\varepsilon}^{i} \ln
$$

and

$$
\mu_{*}(h, \partial \Omega)=\liminf _{\varepsilon \rightarrow+0} \varepsilon^{-(n-h)}\left|\Gamma_{\varepsilon}^{i}\right|_{n}
$$


The interior Bouligand-Minkowski dimension of $\partial \Omega$, denoted by $d_{i}$, is the number

$$
d_{i}=\inf \left\{h \subset \mathbb{R}: \mu^{*}(h, \partial \Omega)=0\right\} .
$$

Obviously $d_{i} \in[n-1, n]$.

An analogous definition can be given for the exterior Bouligand-Minkowski dimension of $\partial \Omega$ by replacing $\Gamma_{\varepsilon}^{i}$ by $\Gamma_{\varepsilon}^{e}$ where

$$
\Gamma_{\varepsilon}^{e}=\{x \notin \bar{\Omega}: \operatorname{dist}(x, \partial \Omega)<\varepsilon\} .
$$

Then

$$
d=\left\{\begin{array}{l}
\max \left(d_{i}, d_{e}\right) \quad \text { if }|\partial \Omega|_{n}=0 \\
n \quad \text { if }|\partial \Omega|_{n} \neq 0
\end{array}\right.
$$

is the Bouligand-Minkowski dimension. This dimension is greater than or equal to the Hausdorff one defined for example in [Fa]. To contradict the Berry conjecture J. Brossard and R. Carmona constructed a domain such that the Bouligand-Minkowski dimension is strictly larger than the Hausdorff one.

In this article we first construct an example of a disconnected open set $\mathscr{Q}$ in $\mathbb{R}^{2}$ for which the second term can be exactly calculated and is of the form announced above $\left(-c p(\ln \lambda) \lambda^{d_{i} / 2}\right)$. We then obtain a lower bound and an upper bound of the same form for a connected open set $\mathscr{O}$ derived from $\mathscr{Q}$. Finally we give some extensions for more general domains $\Omega \subset \mathbb{R}^{n}$ with fractal boundary. We also derive some properties of the $\zeta$-function of our problem.

For the convenience of the reader we introduce now some notations that we use throughout the paper.

For a given positive number $r$ we set

$$
\mathscr{N}_{n}(r)=\#\left\{\left(q_{1}, q_{2}, \ldots, q_{n}\right) \in \mathbb{Z}^{n}: q_{i}>0, q_{1}^{2}+\cdots+q_{n}^{2}<r^{2}\right\} .
$$

We also introduce

$$
\rho_{n}(r):=2^{-n} \omega_{n} r^{n}-\mathcal{N}_{n}(r) .
$$

It is a well-known result-established by Gauss [G]-that there exists a positive constant $\gamma_{n}$, which does not depend on $r$, such that

$$
0<\rho_{n}(r)<\gamma_{n} r^{n-1} \text { for all } r>0 \text {. }
$$

\section{EXAMPLE}

II.A. Open sets and results. Let $s$ be a positive given number satisfying

$$
1+\sqrt{2}<s<3 \text {. }
$$

We consider in $\mathbb{R}^{2}$ the open set $\mathscr{Q}$ (as shown in Figure 1), which consists of a union of open squares. The central square $Q_{0}$ has side 1 . The side of each of the 4 consecutive squares $Q_{1}$ is $s$ times smaller; these squares are "sticked" on the middles of the sides of $Q_{0}$.

We now have $4 \times 3$ "free" sides with length $s^{-1}$; on each middle part of the sides we "stick" again one square $Q_{2}$ with side $s^{-2}$ etc. At the $k$ th step we have

$$
n_{k}=\frac{4}{3} 3^{k}, \quad k \geq 1, \text { and } n_{0}=1
$$

squares $Q_{k}$ with sides $s^{-k}$. 

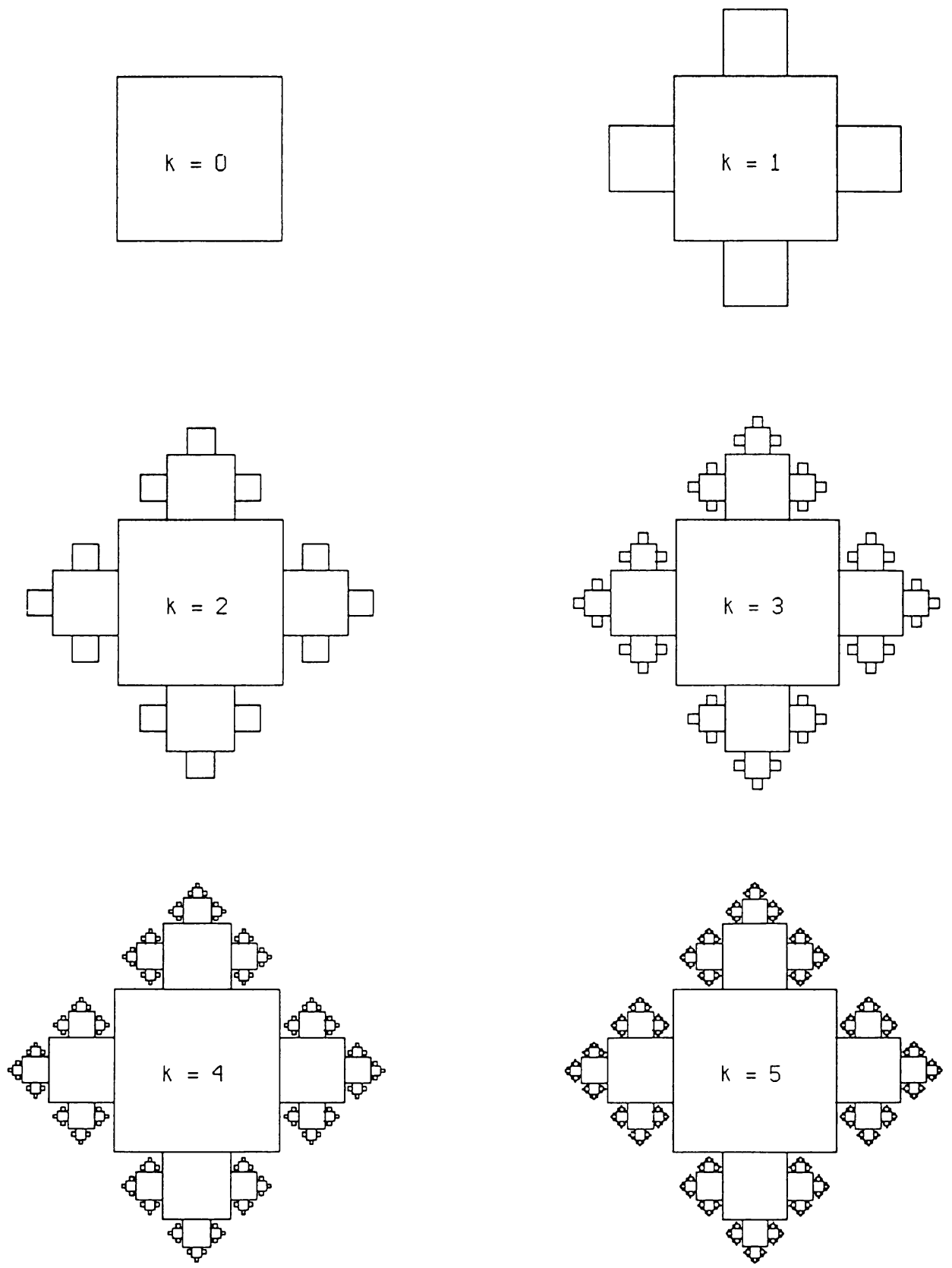

FIGURE 1

We denote by $\mathscr{Q}$ the union of all these squares for $k=0,1,2, \ldots$ Note that $\mathscr{Q}$ is disconnected; moreover it follows from (2.1) that the squares do not overlap and that $\mathscr{Q}$ is with finite measure.

The interior Bouligand-Minkowski dimension $d_{i}$ of $\partial \mathscr{Q}$ is

$$
d_{i}=\frac{\ln 3}{\ln s} \text {. }
$$


This can be derived by a simple calculation, since for a given $\varepsilon>0$

$$
\left|\Gamma_{\varepsilon}^{i}\right|_{2}=\sum_{k=0}^{K} n_{k}\left(4 \varepsilon s^{-k}-4 \varepsilon^{2}\right)+\sum_{k=K+1}^{+\infty} n_{k} s^{-2 k}
$$

where $K$ is such that

$$
s^{-(K+1)}<2 \varepsilon \leq s^{-K} .
$$

Note that it follows from $(2.1)$ that $1<d_{i}<2$.

For this open set $\mathscr{Q}$ we can compute exactly the second term of the asymptotics of the counting function; we have

Proposition 1. As $\lambda$ tends to $+\infty$

$$
N(\lambda, \mathscr{Q})=W(\lambda, \mathscr{Q})-\frac{4}{3}\left(\frac{\sqrt{\lambda}}{\pi}\right)^{d_{i}} p_{2}\left(\frac{\ln \lambda-2 \ln \pi}{2 \ln s}\right)+O(\sqrt{\lambda})
$$

where

$$
p_{2}(y):=\sum_{k=-\infty}^{+\infty} 3^{k-y} \rho_{2}\left(s^{y-k}\right) .
$$

The function $p_{2}$ is well defined, positive, bounded, 1-periodic and left-continuous; moreover the set of its points of discontinuity is dense in $\mathbb{R}$. Here $n=2$ and hence

$$
W(\lambda, \mathscr{Q})=\frac{\lambda|\mathscr{Q}|_{2}}{4 \pi}, \quad \rho_{2}(r)=\frac{\pi}{4} r^{2}-\mathscr{N}_{2}(r)
$$

where $\mathscr{N}_{2}$ is defined by (1.11).

The set $\mathscr{Q}$ being disconnected, we also consider the connected set $\mathscr{O}$, derived from $\mathscr{Q}$ by opening in the middle of each $\partial Q_{k} \cap \partial Q_{k-1}$ a small interval $I_{k}$ with length

$$
\varepsilon_{k}=(100(k !))^{-1} ;
$$

throughout the paper we refer to these $I_{k}$ as "cuts". This connected open set $\mathscr{O}$ has the same Lebesgue measure (in $\mathbb{R}^{2}$ ) as $\mathscr{Q}$ and it also has the same interior Bouligand-Minkowski dimension $d_{i}=\frac{\ln 3}{\ln s}$.

Theorem 1. As $\lambda$ tends to $+\infty$

$$
\begin{aligned}
& -\frac{4}{3}\left(\frac{\sqrt{\lambda}}{\pi}\right)^{d_{i}} p_{2}\left(\frac{\ln \lambda-2 \ln \pi}{2 \ln s}\right)+O(\sqrt{\lambda}) \\
& \quad=N(\lambda, \mathscr{Q})-W(\lambda, \mathscr{Q}) \leq N(\lambda, \mathscr{O})-W(\lambda, \mathscr{O}) \\
& \quad \leq-\frac{4}{3}\left(\frac{\sqrt{\lambda}}{\pi}\right)^{d_{i}} p_{2}\left(\frac{\ln \lambda-2 \ln \pi}{2 \ln s}+o(1)\right)+o\left(\lambda^{d_{i} / 2}\right) .
\end{aligned}
$$

II.B. Proof of (2.6). Since $\mathscr{Q}$ is the union of disjoint squares, we simply have

$$
N(\lambda, \mathscr{Q})=\sum_{k=0}^{+\infty} n_{k} N\left(\lambda, Q_{k}\right)
$$

where $n_{k}$ is given by (2.2). 
On each square $Q_{k}$ the eigenvalues of the Dirichlet Laplacian are $\left(q_{1}^{2}+q_{2}^{2}\right) \pi^{2} s^{2 k}$ where $q_{1}$ and $q_{2}$ are positive integers; hence, by use of (1.11) we have

$$
N\left(\lambda, Q_{k}\right)=N\left(\lambda s^{-2 k}, Q_{0}\right)=\mathscr{N}_{2}\left(\sqrt{\lambda} s^{-k} / \pi\right)
$$

It follows then from (2.8) combined with (2.11) and (2.12) that

$$
\begin{aligned}
N(\lambda, \mathscr{Q})-W(\lambda, \mathscr{Q}) & =\sum_{k=0}^{+\infty} n_{k}\left(\mathscr{N}_{2}\left(\frac{\sqrt{\lambda} s^{-k}}{\pi}\right)-\frac{\lambda}{4 \pi} s^{-2 k}\right) \\
& =-\sum_{k=0}^{+\infty} n_{k} \rho_{2}\left(\frac{\sqrt{\lambda} s^{-k}}{\pi}\right) .
\end{aligned}
$$

Therefore

$$
N(\lambda, \mathscr{Q})-W(\lambda, \mathscr{Q})=A+B
$$

with

$$
A:=-\frac{4}{3} \sum_{k=-\infty}^{+\infty} 3^{k} \rho_{2}\left(\frac{\sqrt{\lambda} s^{-k}}{\pi}\right) \equiv A(\lambda)
$$

and

$$
B:=-\rho_{2}\left(\frac{\sqrt{\lambda}}{\pi}\right)+\frac{4}{3} \sum_{k=-\infty}^{0} 3^{k} \rho_{2}\left(\frac{\sqrt{\lambda} s^{-k}}{\pi}\right) .
$$

We deduce from (2.8) and (2.1) that

$$
|B| \leq \gamma_{2}\left(\frac{\sqrt{\lambda}}{\pi}\right)\left(1+\frac{4}{3} \sum_{j=0}^{+\infty}\left(\frac{s}{3}\right)^{j}\right)=\frac{\gamma_{2}(7-s) \sqrt{\lambda}}{\pi(3-s)} .
$$

Of course the same argument shows that the part corresponding to negative $k$ in the series (2.14) converges. For the other part, corresponding to positive $k$, we simply notice that by $(2.8) \rho_{2}(r) \leq \pi r^{2} / 4$; therefore, by use of $(2.1)$

$$
\sum_{k=1}^{+\infty} 3^{k} \rho_{2}\left(\frac{\sqrt{\lambda} s^{-k}}{\pi}\right) \leq \frac{\lambda}{4 \pi} \sum_{k=1}^{+\infty}\left(\frac{3}{s^{2}}\right)^{k}=\frac{3 \lambda}{4 \pi\left(s^{2}-3\right)} .
$$

It follows from (1.13), (2.1), (2.16) and (2.17) that $A$ given by (2.14) is well defined. We introduce now $y \in \mathbb{R}$ by

$$
s^{y}=\frac{\sqrt{\lambda}}{\pi}, \quad \text { i.e. } \quad y=\frac{\ln \lambda-2 \ln \pi}{2 \ln s} .
$$

This implies that

$$
3^{y}=\left(\frac{\sqrt{\lambda}}{\pi}\right)^{d_{i}} .
$$

Hence, substituting (2.18) and (2.19) in (2.14) we get

$$
A=-\frac{4}{3}\left(\frac{\sqrt{\lambda}}{\pi}\right)^{d_{i}} \sum_{k=-\infty}^{+\infty} 3^{k-y} \rho_{2}\left(s^{y-k}\right) \equiv A(\lambda) .
$$

Finally, (2.6) is a simple consequence of (2.7), (2.13), (2.16), (2.18) and (2.20). 
II.C. The function $p_{2}$. It follows from (2.7) and (2.20) that

$$
A=-\frac{4}{3}\left(\frac{\sqrt{\lambda}}{\pi}\right)^{d_{i}} p_{2}(y)
$$

hence $p_{2}$ is well defined and bounded. Since $k-y=(k+1)-(y+1)$ it is obvious that $p_{2}$ is 1 -periodic.

Moreover, since $\rho_{2}$ is positive, so is $p_{2}$. Obviously $\mathcal{N}_{2}$ is left-continuous, therefore the same remark holds for $p_{2}$. We now notice that

$$
\mathscr{N}_{2}(r)= \begin{cases}0 & \text { when } r \leq \sqrt{2} \\ 1 & \text { when } \sqrt{2}<r \leq \sqrt{5}\end{cases}
$$

so that $r=\sqrt{2}$ is a point of discontinuity for $\mathscr{N}_{2}$; each time that there exist two strictly positive integers $q_{1}$ and $q_{2}$, satisfying $q_{1}^{2}+q_{2}^{2}=r^{2}$, then $r$ is a point of discontinuity for $\mathscr{N}_{2}$. This implies that $p_{2}$ is discontinuous in $y \in \mathbb{R}$ each time there exist three integers $q_{1}, q_{2}, k$, with $q_{1}$ and $q_{2}$ strictly positive, satisfying $q_{1}^{2}+q_{2}^{2}=s^{2(y-k)}$ or equivalently

$$
y=k+\frac{\ln \left(q_{1}^{2}+q_{2}^{2}\right)}{2 \ln s}
$$

In particular this shows that any

$$
y(k, q)=k+\frac{2 \ln q+\ln 2}{2 \ln s}, \quad k \in \mathbb{Z}, q \in \mathbb{Z}_{+},
$$

is a point of discontinuity for $p_{2}$.

Note that $(2.22)$ comes from (2.21) by choosing $q_{1}=q_{2}=q$. Now let $y_{0} \in \mathbb{R}$ be an arbitrary fixed point, and $l$ a positive integer, large enough. Let us denote by $q_{l}$ the maximal natural integer such that

$$
y_{l}:=-l+\frac{2 \ln q_{l}+\ln 2}{2 \ln s} \leq y_{0} .
$$

We have the obvious inequality

$$
\left|y_{0}-y_{l}\right|<\frac{\ln \left(q_{l}+1\right)-\ln q_{l}}{\ln s}=\frac{\ln \left(1+q_{l}^{-1}\right)}{\ln s} .
$$

But the right-hand side of this inequality tends to zero as $l$ tends to $+\infty$ because $q_{l}$ tends to $+\infty$. Therefore the sequence $\left(y_{l}\right)_{l \in \mathbb{N}}$ tends to $y_{0}$ as $l$ tends to $+\infty$, and hence the set of points of discontinuity is dense.

It is useful to compute the Fourier coefficients of the function $p_{2}$ :

$$
c_{m}=\int_{0}^{1} p_{2}(y) \exp (-2 \pi m i y) d y=-\frac{1}{\ln 3+2 \pi m i} \tilde{\zeta}\left(\frac{d_{i}}{2}+\frac{\pi m i}{\ln s}\right),
$$

$m \in \mathbb{Z}$. Here

$$
\tilde{\zeta}(z)=\sum_{q_{1}=1}^{+\infty} \sum_{q_{2}=1}^{+\infty}\left(q_{1}^{2}+q_{1}^{2}\right)^{-z}=\int_{0}^{+\infty} r^{-2 z} d \mathcal{N}_{2}(r)
$$

is the $\zeta$-function of the Dirichlet Laplacian on a square with side $\pi$; this series converges for $\operatorname{Re} z>1$ and $\tilde{\zeta}\left(\left(d_{i} / 2\right)+(\pi m i / \ln s)\right)$ is understood as the result 
of analytic continuation around the pole $z=1$. Alternatively, $\tilde{\zeta}(z)$ for $\operatorname{Re} z \in$ $(1 / 2,1)$ can be defined as

$$
\tilde{\zeta}(z)=\lim _{R \rightarrow+\infty}\left(\sum_{\substack{q_{1}^{2}+q_{2}^{2}<R^{2} \\ q_{1}, q_{2} \in \mathbb{Z} ; q_{1}, q_{2}>0}}\left(q_{1}^{2}+q_{2}^{2}\right)^{-z}+\frac{\pi R^{2-2 z}}{4(z-1)}\right)=-\int_{0}^{+\infty} r^{-2 z} d \rho_{2}(r) .
$$

Numerical results for $s=2.5$ show that $\inf p_{2}(y) \approx 5.67, \sup p_{2}(y) \approx 6.91$. Note that $c_{0} \approx 6.3$ is the mean value of the function $p_{2}$ over a period.

II.D. Proof of (2.10). Of course, since $H_{0}^{1}(\mathscr{O}) \supset H_{0}^{1}(\mathscr{Q})$, we have

$$
N(\lambda, \mathscr{O}) \geq N(\lambda, \mathscr{Q})
$$

It is also obvious that

$$
W(\lambda, \mathscr{O})=W(\lambda, \mathscr{Q}) .
$$

Therefore the left-hand inequality of $(2.10)$ is a combination of Proposition 1 and (2.24) and (2.25).

For having an upper bound we make use of a refinement of the usual "Dirichlet-Neumann bracketing" [CH]. For each $Q_{k}$ let us denote by $\tilde{N}\left(\lambda, Q_{k}\right)$ the number of eigenvalues less than $\lambda$ of the Laplacian defined on $Q_{k}$ with Neumann boundary conditions on the four "cuts" and with Dirichlet boundary conditions elsewhere. We have

$$
N(\lambda, \mathscr{O}) \leq \sum_{k=0}^{+\infty} n_{k} \tilde{N}\left(\lambda, Q_{k}\right)
$$

and hence

$$
N(\lambda, \mathscr{O})-W(\lambda, \mathscr{O}) \leq \sum_{k=0}^{+\infty} n_{k}\left(\tilde{N}\left(\lambda, Q_{k}\right)-W\left(\lambda, Q_{k}\right)\right)=C+D
$$

with

$$
\begin{gathered}
C:=\sum_{k=0}^{+\infty} n_{k}\left(\tilde{N}\left(\lambda, Q_{k}\right)-N\left(\lambda, Q_{k}\right)\right) \\
D:=\sum_{k=0}^{+\infty} n_{k}\left(N\left(\lambda, Q_{k}\right)-W\left(\lambda, Q_{k}\right)\right) \equiv N(\lambda, \mathscr{Q})-W(\lambda, \mathscr{Q}) .
\end{gathered}
$$

An estimate for $C$ is obtained through the use of the following result which will be proved in the next subsection.

\section{Proposition 2.}

$$
\tilde{N}\left(\lambda, Q_{k}\right) \leq N\left(\lambda\left(1+\omega_{k}\right), Q_{k}\right)
$$

where $\omega_{k}$ is some positive number which depends only on $k$ and which tends to zero as $k$ tends to $+\infty$.

Let us finish the proof of Theorem 1 by finding an upper bound for $C$.

Consider a positive integer $J$ and set

$$
K(\lambda)=E[(\ln \lambda) /(2 \ln s)]
$$


where $E[\cdot]$ denotes the entire part. Here and below $\lambda>0$ is assumed to be sufficiently large. Note that (2.29) implies

$$
\begin{gathered}
\sqrt{\lambda} / s<s^{K(\lambda)} \leq \sqrt{\lambda}, \\
\lambda^{d_{i} / 2} / 3<3^{K(\lambda)} \leq \lambda^{d_{i} / 2} .
\end{gathered}
$$

We now split $C$ into two parts

$$
C=C_{1}+C_{2}
$$

with

$$
\begin{aligned}
& C_{1}=\sum_{\substack{|k-K(\lambda)|>J \\
k \geq 0}} n_{k}\left(\tilde{N}\left(\lambda, Q_{k}\right)-N\left(\lambda, Q_{k}\right)\right), \\
& C_{2}=\sum_{\substack{|k-K(\lambda)| \leq J \\
k \geq 0}} n_{k}\left(\tilde{N}\left(\lambda, Q_{k}\right)-N\left(\lambda, Q_{k}\right)\right) .
\end{aligned}
$$

We remark that in view of (2.30) for $k>K(\lambda)+J$ we have $2 \pi^{2} s^{2 k}>\lambda\left(1+\omega_{k}\right)$; hence, by $[\mathrm{CH}]$ and Proposition 2 for these $k$

$$
0 \leq N\left(\lambda, Q_{k}\right) \leq \tilde{N}\left(\lambda, Q_{k}\right) \leq N\left(\lambda\left(1+\omega_{k}\right), Q_{k}\right)=0 .
$$

Therefore, by (2.33) and (2.35), $C_{1}$ is reduced to

$$
C_{1}=\sum_{k=0}^{K(\lambda)-J-1} n_{k}\left(\tilde{N}\left(\lambda, Q_{k}\right)-N\left(\lambda, Q_{k}\right)\right) .
$$

For each square $Q_{k}$ we know [CH] that there exists a constant $\gamma>0$, which does not depend on $\lambda$ or $k$, such that

$$
\begin{aligned}
& W\left(\lambda, Q_{k}\right)-\gamma\left(1+\sqrt{\lambda} s^{-k}\right) \leq N\left(\lambda, Q_{k}\right) \\
& \quad \leq \widetilde{N}\left(\lambda, Q_{k}\right) \leq N_{1}\left(\lambda, Q_{k}\right) \leq W\left(\lambda, Q_{k}\right)+\gamma\left(1+\sqrt{\lambda} s^{-k}\right)
\end{aligned}
$$

where $N_{1}\left(\lambda, Q_{k}\right)$ denotes the number of eigenvalues less than $\lambda$ for the Neumann Laplacian on $Q_{k}$.

As a consequence of $(2.36),(2.2)$ and (2.37) we have

$$
\begin{aligned}
C_{1} & <\frac{8 \gamma}{3} \sum_{k=-\infty}^{K(\lambda)-J-1} 3^{k}\left(1+\sqrt{\lambda} s^{-k}\right) \\
& <\frac{8 \gamma}{3-s}\left[3^{K(\lambda)-J-1}+\sqrt{\lambda}\left(\frac{3}{s}\right)^{K(\lambda)-J-1}\right] ;
\end{aligned}
$$

with the help of (2.30) and (2.31) this formula transforms into

$$
C_{1}<\frac{8 \gamma(1+s) \lambda^{d_{i} / 2}}{3-s}\left(\frac{s}{3}\right)^{J+1}
$$

We consider now $C_{2}$. By Proposition 2

$$
\begin{aligned}
C_{2} & \leq \sum_{\substack{|k-K(\lambda)| \leq J \\
k \geq 0}} n_{k}\left(N\left(\lambda\left(1+\omega_{k}\right), Q_{k}\right)-N\left(\lambda, Q_{k}\right)\right) \\
& \leq \sum_{\substack{|k-K(\lambda)| \leq J \\
k \geq 0}} n_{k}\left(N\left(\lambda(1+\omega(\lambda, J)), Q_{k}\right)-N\left(\lambda, Q_{k}\right)\right)
\end{aligned}
$$


where

(2.40)

$$
\omega(\lambda, J):=\max _{|k-K(\lambda)| \leq J} \omega_{k} .
$$

It follows from Proposition 2 and from (2.29) that

$$
\omega(\lambda, J) \rightarrow+0 \text { as } \lambda \rightarrow+\infty
$$

(of course the rate at which $\omega(\lambda, J)$ tends to +0 depends on $J$ ).

We can rewrite the inequality (2.39) in the form

$$
C_{2} \leq F_{1}+F_{2}-F_{3}
$$

where

$$
F_{1}=\sum_{\substack{|k-K(\lambda)| \leq J \\ k \geq 0}} n_{k}\left(W\left(\lambda(1+\omega(\lambda, J)), Q_{k}\right)-W\left(\lambda, Q_{k}\right)\right),
$$

$$
F_{2}=\sum_{\substack{|k-K(\lambda)| \leq J \\ k \geq 0}} n_{k} \rho_{2}\left(\frac{\sqrt{\lambda} s^{-k}}{\pi}\right)
$$

$$
F_{3}=\sum_{\substack{|k-K(\lambda)| \leq J \\ k \geq 0}} n_{k} \rho_{2}\left(\frac{\sqrt{\lambda(1+\omega(\lambda, J))} s^{-k}}{\pi}\right) .
$$

From (2.43), (2.2), (2.8), (2.30) and (2.31) we deduce

$$
F_{1}<\frac{s^{4} \omega(\lambda, J) \lambda^{d_{i} / 2}}{3 \pi\left(s^{2}-3\right)}\left(\frac{s^{2}}{3}\right)^{J}
$$

For estimating $F_{2}$ and $F_{3}$ we extend the summation in (2.44) and (2.45) to all $k \in \mathbb{Z}$ with $n_{k}=4 \cdot 3^{k-1}$ and by (2.14) arrive at

$$
\begin{gathered}
F_{2}<-A(\lambda), \\
F_{3}=-A(\lambda(1+\omega(\lambda, J)))-\frac{4}{3} \sum_{k=-\infty}^{K(\lambda)-J-1} 3^{k} \rho_{2}\left(\frac{\sqrt{\lambda(1+\omega(\lambda, J))} s^{-k}}{\pi}\right) \\
-\frac{\lambda(1+\omega(\lambda, J))}{3 \pi} \sum_{k=K(\lambda)+J+1}^{+\infty}\left(\frac{3}{s^{2}}\right)^{k} .
\end{gathered}
$$

By use of (2.20) and (2.7) we derive from (2.47)

$$
F_{2}<\frac{4}{3}\left(\frac{\sqrt{\lambda}}{\pi}\right)^{d_{i}} p_{2}\left(\frac{\ln \lambda-2 \ln \pi}{2 \ln s}\right) .
$$

Handling (2.48) analogously but with the use of (1.13), (2.30) and (2.31) we obtain

$$
\begin{aligned}
-F_{3}< & -\frac{4}{3}\left(\frac{\sqrt{\lambda(1+\omega(\lambda, J))}}{\pi}\right)^{d_{i}} p_{2}\left(\frac{\ln (\lambda(1+\omega(\lambda, J)))-2 \ln \pi}{2 \ln s}\right) \\
& +\frac{4 s \gamma_{2} \sqrt{1+\omega(\lambda, J)} \lambda^{d_{i} / 2}}{\pi(3-s)}\left(\frac{s}{3}\right)^{J+1}+\frac{s^{2}(1+\omega(\lambda, J)) \lambda^{d_{i} / 2}}{\pi\left(s^{2}-3\right)}\left(\frac{3}{s^{2}}\right)^{J} .
\end{aligned}
$$


Up to this moment the number $J$ was fixed. But from now on we assume that $J$ is a function of $\lambda$ such that

$$
J(\lambda) \rightarrow+\infty \text { and } \omega(\lambda, J(\lambda))\left(s^{2} / 3\right)^{J(\lambda)} \rightarrow+0 \text { as } \lambda \rightarrow+\infty .
$$

Of course this can always be achieved in view of (2.41). Bringing together formulas (2.1), (2.6), (2.26), (2.28), (2.32), (2.38), (2.42), (2.46), (2.49), (2.50) and making use of (2.51) we finally obtain the right-hand inequality (2.10).

II.E. Proof of Proposition 2. We intend in this section to establish an upper bound for $\widetilde{N}\left(\lambda, Q_{k}\right)$; let us recall that $\widetilde{N}\left(\lambda, Q_{k}\right)$ is the counting function of the Laplacian on $Q_{k}$ with Neumann boundary conditions on the "cuts" and Dirichlet ones elsewhere. But it follows from (2.9) that for a given $Q_{k}$ the "cuts" are with size $\varepsilon_{k}$ (for one of them) and $\varepsilon_{k+1}$ (for three of them). Hence, as a consequence of the inclusions of Sobolev spaces, we may write

$$
\tilde{N}\left(\lambda, Q_{k}\right) \leq \tilde{N}\left(\lambda, Q_{k}, \varepsilon_{k}\right)
$$

where $\tilde{N}\left(\lambda, Q_{k}, \varepsilon_{k}\right)$ denotes the number of eigenvalues less than $\lambda$ for the Laplacian defined on $Q_{k}$ with Dirichlet boundary conditions everywhere but on the four enlarged "cuts" where we consider the Neumann boundary conditions; these enlarged "cuts" are all with the same size $\varepsilon_{k}$, and, as previously, they are in the middle of the sides of the square.

We can now change the scale so that we have to study the eigenvalues $\tilde{\lambda}_{j}(Q, \eta)$ of the Laplacian defined on a unit square $Q$ with Neumann boundary conditions on "cuts" with length $\eta=s^{k} \varepsilon_{k}$ and Dirichlet ones elsewhere; in the following we denote by $\widetilde{H}(Q, \eta)$ the variational space associated with this problem and by $\widetilde{N}(\lambda, Q, \eta)$ the corresponding counting function. We have

$$
\tilde{\lambda}_{j}\left(Q_{k}, \varepsilon_{k}\right)=s^{2 k} \tilde{\lambda}_{j}\left(Q, s^{k} \varepsilon_{k}\right)
$$

or equivalently

$$
\tilde{N}\left(\lambda, Q_{k}, \varepsilon_{k}\right)=\tilde{N}\left(\lambda s^{-2 k}, Q, s^{k} \varepsilon_{k}\right) .
$$

Remark 1. It follows from (2.9) that the length of the rescaled "cuts" $\eta=s^{k} \varepsilon_{k}$ tends to zero as $k$ tends to $+\infty$.

By inclusion of the variational spaces

$$
H_{0}^{1}(Q) \subset \widetilde{H}\left(Q, \eta_{1}\right) \subset \widetilde{H}\left(Q, \eta_{2}\right) \subset H^{1}(Q)
$$

for $0<\eta_{1} \leq \eta_{2}<1$, we deduce that for any integer $j \geq 1$

$$
\lambda_{j}^{N}(Q) \leq \tilde{\lambda}_{j}\left(Q, \eta_{2}\right) \leq \tilde{\lambda}_{j}\left(Q, \eta_{1}\right) \leq \lambda_{j}^{D}(Q),
$$

where $\lambda_{j}^{D}(Q)$ (resp. $\left.\lambda_{j}^{N}(Q)\right)$ denotes the $j$ th eigenvalue of the Dirichlet (resp. Neumann) Laplacian on $Q$. Thus by (2.56), as $\eta$ decreases to $+0, \tilde{\lambda}_{j}(Q, \eta)$ increases to

$$
\lim _{\eta \rightarrow+0} \tilde{\lambda}_{j}(Q, \eta):=\tilde{\lambda}_{j}(Q,+0) \leq \lambda_{j}^{D}(Q) .
$$

We shall show now that, in fact, (2.57) is an equality:

$$
\tilde{\lambda}_{j}(Q,+0)=\lambda_{j}^{D}(Q) \text {. }
$$

For simplicity we shall prove (2.58) for $j=1$. 
Denote by $\varphi_{1}(\eta) \in H^{1}(Q)$ the eigenfunction associated with $\tilde{\lambda}_{1}(Q, \eta)$ and normalized by the condition $\left\|\varphi_{1}(\eta)\right\|_{L_{2}(Q)}=1$; obviously $\left\|\varphi_{1}(\eta)\right\|_{H^{1}(Q)}^{2}=$ $\tilde{\lambda}_{1}(Q, \eta)+1$. For any monotonic sequence of positive numbers $\eta_{p}$ tending to zero as $p \rightarrow+\infty$ we have a sequence $\varphi_{1}\left(\eta_{p}\right)$ bounded in $L_{2}(Q)$ as well as in $H^{1}(Q)$. Since the embedding of $H^{1}(Q)$ into $L_{2}(Q)$ is compact there exists a subsequence of $\varphi_{1}\left(\eta_{p}\right)$ (still denoted by $\varphi_{1}\left(\eta_{p}\right)$ below) which converges to some $\psi \in H^{1}(Q)$, the convergence being strong in $L_{2}(Q)$ and weak in $H^{1}(Q)$. Obviously

$$
\begin{gathered}
\|\psi\|_{L_{2}(Q)}=\lim _{p \rightarrow+\infty}\left\|\varphi_{1}\left(\eta_{p}\right)\right\|_{L_{2}(Q)}=1, \\
\|\psi\|_{H^{1}(Q)}^{2} \leq \lim _{p \rightarrow+\infty}\left\|\varphi_{1}\left(\eta_{p}\right)\right\|_{H^{1}(Q)}^{2}=\tilde{\lambda}_{1}(Q,+0)+1, \\
\psi \subset \bigcap_{\eta>0} \tilde{H}(Q, \eta) .
\end{gathered}
$$

But (2.61) means that the trace $\left.\psi\right|_{\partial Q}$ is zero almost everywhere $\left(\left.\psi\right|_{\partial Q} \equiv 0\right.$ except on four points which are the middles of the four sides) hence $\psi \in H_{0}^{1}(Q)$. By the min max principle for the Dirichlet problem we have in view of (2.59)

$$
\|\psi\|_{H^{1}(Q)}^{2} \geq \lambda_{1}^{D}(Q)+1 .
$$

Formula (2.58) follows from (2.57), (2.60) and (2.62).

Of course the above argument can be extended to the $j$ th eigenvalues by dealing with $j$-dimensional spaces of functions.

Let us show now that uniformly over $\lambda>0$

$$
\widetilde{N}(\lambda, Q, \eta) \leq N(\lambda(1+\omega(\eta)), Q)
$$

where

$$
\omega(\eta) \rightarrow+0 \text { as } \eta \rightarrow+0
$$

recall that $N(\cdot, Q)$ is the counting function of the Dirichlet problem on the unit square. Let $\delta>0$ be an arbitrary number. By $[\mathrm{CH}]$ we have

$$
\begin{gathered}
\left|\tilde{N}(\lambda, Q, \eta)-(4 \pi)^{-1} \lambda\right| \leq \tilde{\gamma} \sqrt{\lambda}, \\
\left|N(\lambda, Q)-(4 \pi)^{-1} \lambda\right| \leq \tilde{\gamma} \sqrt{\lambda},
\end{gathered}
$$

where $\tilde{\gamma}>0$ does not depend on $\lambda>0$ or $\eta \in(0,1 / 2]$. Set

$$
\Lambda(\delta)=\left(4 \pi \tilde{\gamma} \delta^{-1}(1+\sqrt{1+\delta})\right)^{2} .
$$

It follows from (2.65)-(2.67) that for $\lambda \geq \Lambda(\delta)$

$$
\tilde{N}(\lambda, Q, \eta) \leq N(\lambda(1+\delta), Q) .
$$

Note now that the number of eigenvalues $\tilde{\lambda}_{j}(Q, \eta)$ and $\lambda_{j}^{D}(Q)$ on $(0, \Lambda(\delta)]$ is finite (not more than the number of $\lambda_{j}^{N}(Q)$ on this segment). Using the fact that these $\tilde{\lambda}_{j}(Q, \eta)$ tend to $\lambda_{j}^{D}(Q)$ as $\eta$ tends to +0 (see (2.58)) we obtain (2.68) for $\lambda<\Lambda(\delta)$ and $\eta<\tilde{\eta}(\Lambda(\delta))$. As $\delta>0$ is arbitrary, formulas (2.63) and (2.64) follow from (2.68).

Combining formulas (2.52), (2.54), (2.63), Remark 1 and making an inverse rescaling $N(\lambda, Q)=N\left(\lambda s^{2 k}, Q_{k}\right)$ we obtain Proposition 2 with $\omega_{k}=\omega\left(s^{k} \varepsilon_{k}\right)$. 
II.F. The $\zeta$-function. For the Dirichlet Laplacian on a bounded open set $\Omega$ with eigenvalues $\lambda_{j}(\Omega)$ we define as usual the $\zeta$-function of the problem

$$
\zeta(z, \Omega)=\sum_{j=1}^{+\infty} \lambda_{j}^{-z}(\Omega)=\int_{0}^{+\infty} \lambda^{-z} d N(\lambda, \Omega) .
$$

Substituting (2.6) into (2.69) we immediately obtain the following result.

Proposition 3. For our example $\mathscr{Q}$ the function $\zeta(z, \mathscr{Q})$ is meromorphic in the complex half-plane $\operatorname{Re} z>1 / 2$ with simple poles at $z=1$ and $z=z_{m}:=$ $d_{i} / 2+(\pi m i) /(\ln s), m \in \mathbb{Z}$. The corresponding residues are

$$
\operatorname{Res}(1, \mathscr{Q})=|\mathscr{Q}| / 4 \pi, \quad \operatorname{Res}\left(z_{m}, \mathscr{Q}\right)=-4 c_{m} z_{m} / 3 \pi^{2 z_{m}},
$$

where $c_{m}$ are the Fourier coefficients of the function $p_{2}$, see (2.23).

It can be shown that Proposition 3 holds also for the domain $\mathscr{O}$ but unfortunately this fact does not follow immediately from (2.10). Proving Proposition 3 for the domain $\mathscr{O}$ demands more delicate estimates which lie beyond the scope of this work.

These results can be related to some new conjectures in [L2] or [LP].

II.G. Numerical results show that whereas for the smooth case the two-term asymptotics usually start working effectively form the very beginning of the spectrum [V1] the fractal two-term asymptotics become effective only for very big $\left(\lambda \geq 10^{6}\right)$ values of $\lambda$. This is illustrated by the following table, containing results for the spectrum of the Dirichlet Laplacian on the open set $\mathscr{Q}(s=2.5)$ :

\begin{tabular}{cccccc} 
& $\lambda=10^{2}$ & $\lambda=10^{4}$ & $\lambda=10^{6}$ & $\lambda=10^{8}$ & $\lambda=10^{10}$ \\
\hline$N(\lambda, \mathscr{Q})$ & 6 & 1440 & 171289 & 17635990 & 1773236170 \\
\hline$W_{2}(\lambda, \mathscr{Q})$ & -16.2 & 1216 & 169080 & 17614000 & 1773000000 \\
\hline$W(\lambda, \mathscr{Q})$ & 17.8 & 1775 & 177520 & 17752000 & 1775200000 \\
\hline
\end{tabular}

Here

$$
W_{2}(\lambda, \mathscr{Q}):=W(\lambda, \mathscr{Q})-\frac{4}{3}\left(\frac{\sqrt{\lambda}}{\pi}\right)^{d_{i}} p_{2}\left(\frac{\ln \lambda-2 \ln \pi}{2 \ln s}\right)
$$

is the fractal two-term asymptotics.

Numerical estimates of $N(\lambda, \mathscr{Q})$ and of $p_{2}(y)$ have been done by Hervé Innocenti and Dominique Pairault, "élèves" a l'Ecole Nationale Supérieure de l'Aeronautique et de l'Espace ("Sup Aero"), Toulouse, France, where the first author works part-time as a professor.

\section{EXTENSIONS}

As announced in [FV], we shall give a very precise lower bound for the counting function of the Dirichlet (or Neumann) Laplacian on a general domain $\Omega \subset \mathbb{R}^{n}$. Indeed, upper bounds can also be obtained by use of methods from $[\mathrm{FM}, \mathrm{Mt} 1, \ldots]$, but since they are not as precise as Theorem 1 and for brevity we restrict ourselves to the lower bound. 
Theorem 2. Let $\Omega$ be a bounded domain in $\mathbb{R}^{n}$ for which the boundary $\partial \Omega$ is with interior Bouligand-Minkowski dimension $d_{i} \in(n-1, n)$; we assume that

$$
\mu^{*}\left(d_{i}, \partial \Omega\right)<+\infty
$$

where $\mu^{*}\left(d_{i}, \partial \Omega\right)$ is defined by (1.8). Then, for $\lambda \rightarrow+\infty$

$$
\begin{aligned}
& N(\lambda, \Omega)-W(\lambda, \Omega) \\
& \quad \geq-(4 n)^{\left(n-d_{i}\right) / 2} \mu^{*}\left(d_{i}, \partial \Omega\right)\left(\frac{\sqrt{\lambda}}{\pi}\right)^{d_{i}} f_{n}\left(\frac{\ln \lambda-2 \ln \pi}{2 \ln 2}\right)+o\left(\lambda^{d_{i} / 2}\right)
\end{aligned}
$$

where

$$
f_{n}(t)=\sum_{k=-\infty}^{+\infty} 2^{d_{i}(k-t)} \rho_{n}\left(2^{t-k}\right) .
$$

The function $f_{n}$ is well defined, positive, bounded, 1-periodic and left-continuous; moreover the set of its points of discontinuity is dense in $\mathbb{R}$.

Proof of Theorem 2. We consider a "Whitney covering" of $\mathbb{R}^{n}$. For each integer $k \in \mathbb{N}$, we consider a tessalation of $\mathbb{R}^{n}$ into congruent and nonoverlapping open cubes $\left(Q_{\zeta_{k}}\right), \zeta_{k} \in \mathbb{Z}^{n}$, with sides $\eta_{k}=2^{-k}$. We define by induction

$$
\begin{aligned}
& A_{0}=\left\{\zeta_{0} \in \mathbb{Z}^{n}: Q_{\zeta_{0}} \subset \Omega\right\} ; \quad \Omega_{0}^{\prime}=\bigcup_{\zeta_{0} \in A_{0}} Q_{\zeta_{0}} ; \quad \Omega_{0}^{\prime \prime}=\Omega \backslash \overline{\Omega_{0}^{\prime}} ; \\
& A_{1}=\left\{\zeta_{1} \in \mathbb{Z}^{n}: Q_{\zeta_{1}} \subset \Omega_{0}^{\prime \prime}\right\} ; \quad \Omega_{1}^{\prime}=\Omega_{0}^{\prime} \cup\left(\bigcup_{\zeta_{1} \in A_{1}} Q_{\zeta_{1}}\right) ; \quad \Omega_{1}^{\prime \prime}=\Omega \backslash \overline{\Omega_{1}^{\prime}} ; \\
& \cdots \ldots . . \\
& A_{k}=\left\{\zeta_{k} \in \mathbb{Z}^{n}: Q_{\zeta_{k}} \subset \Omega_{k-1}^{\prime \prime}\right\} ; \quad \Omega_{k}^{\prime}=\Omega_{k-1}^{\prime} \cup\left(\bigcup_{\zeta_{k} \in A_{k}} Q_{\zeta_{k}}\right) ; \quad \Omega_{k}^{\prime \prime}=\Omega \backslash \overline{\Omega_{k}^{\prime}} ;
\end{aligned}
$$

In other words, we include in $\Omega$ all the cubes with side $\eta_{0}=1$ that we can; we denote by $A_{0}$ the set of their indices, so that we have $n_{0}=\left(\# A_{0}\right)$ such cubes. In the remaining part $\Omega_{0}^{\prime \prime}$, which is "near" the boundary, we include $n_{1}=\left(\# A_{1}\right)$ cubes with side $\eta_{1}=\eta_{0} / 2$ etc. We make the following remark, as in [LF1]:

Remark 2. $\Omega_{k}^{\prime \prime} \subset \Gamma_{\nu \eta_{k}}^{i}$, where $\nu=n^{1 / 2}$.

The "remaining part" $\Omega_{k}^{\prime \prime}$ at the $k$ th step is included in the "Minkowski sausage" with width $\nu \eta_{k}$; therefore, by (1.8) and (3.1), we have

$$
n_{k+1} \leq \mu^{*}\left(d_{i}, \partial \Omega\right) \nu^{n-d_{i}} 2^{d_{i} k+n}(1+o(1)) \quad \text { as } k \rightarrow+\infty \text {. }
$$

Since

$$
N(\lambda, \Omega) \geq \sum_{k=0}^{+\infty} n_{k} N\left(\lambda, Q_{k}\right),
$$

$\left(Q_{k}\right.$ is a cube with side $\left.\eta_{k}\right)$ we are lead to calculations analogous to those handled in §II.B. It follows from (1.11), (1.12) and (3.5) that

$$
W(\lambda, \Omega)-N(\lambda, \Omega) \leq \sum_{k=0}^{+\infty} n_{k} \rho_{n}\left(\frac{\sqrt{\lambda} 2^{-k}}{\pi}\right) .
$$


Hence, by (3.4)

$$
\begin{aligned}
& W(\lambda, \Omega)-N(\lambda, \Omega) \\
& \quad \leq \mu^{*}\left(d_{i}, \partial \Omega\right)(2 \nu)^{n-d_{i}} \sum_{k=0}^{+\infty} 2^{d_{i} k}(1+o(1)) \rho_{n}\left(\frac{\sqrt{\lambda} 2^{-k}}{\pi}\right) .
\end{aligned}
$$

Since $d_{i}<n$ by hypothesis, the series (3.6) converges by use of the obvious inequality

$$
0<\rho_{n}(r) \leq 2^{-n} \omega_{n} r^{n}
$$

(consequence of (1.12)). By (1.13) and since $d>n-1$ we have

$$
\sum_{k=-\infty}^{-1} 2^{d_{i} k} \rho_{n}\left(\frac{\sqrt{\lambda} 2^{-k}}{\pi}\right) \leq C \lambda^{(n-1) / 2} \sum_{j=1}^{+\infty}\left(\frac{2^{n-1}}{2^{d_{i}}}\right)^{j} \leq C^{\prime} \lambda^{(n-1) / 2} .
$$

Moreover, using (1.13) for $k \leq(\ln \lambda) /(2 \ln 2)$ and (3.7) for $k>(\ln \lambda) /(2 \ln 2)$ we obtain

$$
\sum_{k=0}^{+\infty} 2^{d_{i} k} o(1) \rho_{n}\left(\frac{\sqrt{\lambda} 2^{-k}}{\pi}\right)=o\left(\lambda^{d_{i} / 2}\right) \text { as } \lambda \rightarrow+\infty
$$

Thus, by setting $\sqrt{\lambda} / \pi=2^{t}$, we derive from (3.3), (3.6), (3.8) and (3.9)

$$
W(\lambda, \Omega)-N(\lambda, \Omega) \leq\left(\frac{\sqrt{\lambda}}{\pi}\right)^{d_{i}} \mu^{*}\left(d_{i}, \partial \Omega\right)(2 \nu)^{n-d_{i}} 2^{d_{i} t} f_{n}(t)+o\left(\lambda^{d_{i} / 2}\right)
$$

which is the estimate (3.2) with $t=\ln (\sqrt{\lambda} / \pi) / \ln 2$.

The proof of the properties of the function $f_{n}$ can be handled exactly as those of the function $p_{2}$.

Remark 3. Our method applied to the Brossard-Carmona open set $D$ with a positive integer parameter $a$ gives

$$
N(\lambda, D)=W(\lambda, D)-\frac{4}{5}\left(\frac{\sqrt{\lambda}}{\pi}\right)^{d_{i}} q\left(\frac{\ln \lambda-2 \ln \pi}{2 \ln 3 a}\right)+O(\sqrt{\lambda})
$$

where

$$
q(y)=\sum_{k=-\infty}^{+\infty}\left(5 a^{2}\right)^{k-y} \rho_{2}\left((3 a)^{y-k}\right)
$$

is positive, bounded, left-continuous, 1-periodic and with a dense set of points of discontinuity. This explains of course the fact that the authors [BC] did not find the same upper and lower bounds for the second asymptotic term of the "partition function" $Z(t)=\int_{0}^{+\infty} \exp (-\lambda t) d N(\lambda), t \rightarrow+0$.

Remark 4. As in [BC] we have in our examples $\Omega=\mathscr{Q}$ and $\Omega=\mathscr{O}$ strict inequalities

$$
0<\mu_{*}\left(d_{i}, \partial \Omega\right)<\mu^{*}\left(d_{i}, \partial \Omega\right)<+\infty
$$

so the boundary is not in fact (internally) $d_{i}$-Minkowski measurable. However an equality $\mu_{*}\left(d_{i}, \partial \Omega\right)=\mu^{*}\left(d_{i}, \partial \Omega\right)$ can always be easily achieved (see also [BC, p. 106]) by extracting a specially chosen infinite sequence of isolated points 
from $\Omega$; this will not change the spectrum but will change $\mu_{*}\left(d_{i}, \partial \Omega\right)$ and $\mu^{*}\left(d_{i}, \partial \Omega\right)$. For example, set the origin of our Cartesian coordinate system in the center of $Q_{0}$ and extract from $\mathscr{O}$ (without changing the spectrum!) the sequence of points

$$
A_{m, n}:=\left(\frac{2}{m}, \frac{n}{m !} F\left(\frac{1}{m}\right)\right), \quad m=1,2, \ldots, n=1,2, \ldots, m !,
$$

where

$$
F(z)=\left[\left(z \frac{d}{d z}\right)^{2}-1\right] G(z)+c z^{3-2 d_{i}} \geq 0,
$$

$4 \sqrt{\varepsilon} G(\sqrt{\varepsilon})=\left|\Gamma_{\varepsilon}^{i}\right|_{2}$, and $\left|\Gamma_{\varepsilon}^{i}\right|_{2}$ is defined by formulas (2.4), (2.5), (2.2). Then we obtain (by a straightforward calculation) $\mu_{*}\left(d_{i}, \partial \Omega\right)=\mu^{*}\left(d_{i}, \partial \Omega\right)$. This observation combined with Theorem 1 disproves the modified Berry conjecture as formulated in [L1].

Remark 5. From the physical point of view our example of the "fractal drum" (2) has a certain defect in the sense that part of the boundary of $\Omega$ is "interior" (in some places it separates parts of $\Omega$ ). However we can make all the boundary "exterior" (i.e. separating $\Omega$ and $\mathbb{R}^{2} \backslash \bar{\Omega}$ ) by expanding the lines $\partial \mathscr{Q}_{k} \cap \partial \mathscr{Q}_{k+1}$ into narrow rectangles of width $(100(k !))^{-1}$. Estimates for the counting function of the Dirichlet Laplacian on such a domain are similar to that for $\mathscr{O}$ and the second asymptotic term is the same.

Remark 6. Sometimes the Weyl law plus smooth corrections is regarded as the asymptotic expansion of the smoothed (in some sense) counting function; under such an approach, the periodic function $p_{2}(y)$ in the second asymptotic term might be replaced by a corresponding transform, e.g. its average value $c_{0}$ which was defined in (2.23).

\section{ACKNOWLEDGMENTS}

J. Fleckinger is grateful to the French Ministère des Affaires Etrangères and to the Institute for Problems in Mechanics of the Russian Academy of Sciences and D. G. Vassiliev is grateful to UFR MIG, Université Toulouse 3. These organizations made this collaboration possible. The authors also thank the British Council, the Mathematical Institute of Oberwolfach (Germany) and the organizers of the Seminar on Semi-Classical Methods (Université Paris 13). Moreover the first author thanks W. D. Evans for the "gathering" on this topic organized at the University of Cardiff (Great Britain) and the second author thanks $J$. Brüning for organizing the International Conference " 25 Years of Microlocal Analysis" (Irsee, Germany).

\section{REFERENCES}

[Be] M. V. Berry, Some geometric aspects of wave motion: wavefront dislocations, diffraction catastrophes, diffractals, Geometry of the Laplace Operator, Proc. Sympos. Pure Math., vol. 36, Amer. Math. Soc., Providence, R.I., 1980, pp. 13-38.

[BS] M. Birman and M. Solomjak, On the principal term of the spectral asymptotics for nonsmooth elliptic problems, Funktsional Anal. i Prilozhen. 4 (1970), no. 4, 1-13; English transl. in Funct. Anal. Appl. 4 (1970). 
[BC] J. Brossard and R. Carmona, Can one hear the dimension of a fractal?, Comm. Math. Phys. 104 (1986), 103-122.

[CH] R. Courant and D. Hilbert, Methods of mathematical physics, Vol. 1, English transl., Interscience, New York, 1953.

[DG] J. J. Duistermaat and V. W. Guillemin, The spectrum of positive elliptic operators and periodic bicharacteristics, Invent. Math. 29 (1975), 39-79.

[Fa] K. J. Falconer, The geometry of fractal sets, Cambridge Univ. Press, 1985.

[FM] J. Fleckinger and G. Métivier, Théorie spectrale des opérateurs uniformément elliptiques sur quelques ouverts irréguliers, C.R. Acad. Sci. Paris Sér. A 276 (1973), 913-916.

[FV] J. Fleckinger and D. Vasil'ev, Tambour fractal: exemple d'une formule asymptotique a deux termes pour la "fonction de comptage", C.R. Acad. Sci. Paris Sér. I Math. 311 (1990), 867-872.

[G] C. F. Gauss, Disquisitiones arithmeticae, Leipzig, 1801.

[Iv1] V. Ya. Ivrii, On the second term of the spectral asymptotics for the Laplace-Beltrami operator on a manifold with a boundary, Funktsional Anal. i Prilozhen 14 (1980), no. 2, 25-35; English transl. in Funct. Anal. Appl. 14 (1980).

[Iv2] _ Precise spectral asymptotics for elliptic operators acting in fiberings over manifolds with boundary, Lecture Notes in Math., vol. 1100, Springer-Verlag, Berlin, 1984.

[L1] M. L. Lapidus, Fractal drum, inverse spectral problems for elliptic operators and a partial resolution of the Weyl-Berry conjecture, Trans. Amer. Math. Soc. 325 (1991), 465-529.

[L2] _ Spectral and fractal geometry: from the Weyl-Berry conjecture for the vibrations of fractal drums to the Riemann zeta-function, Differential Equations and Mathematical Physics, Proc. UAB Internat. Conf. on Math. Phys. and Differential Equations held in Birmingham in March 1990 (C. Bennewitz et al., eds.), Academic Press, New York, 1992, pp. 151-182.

[LF1] M. L. Lapidus and J. Fleckinger, The vibrations of a fractal drum, Differential Equations, Lecture Notes in Pure and Appl. Math., Dekker, New York and Basel, 1989, pp. 423-436.

[LF2] _- Tambour fractal: vers une résolution de la conjecture de Weyl-Berry pour les valeurs propres du laplacien, C.R. Acad. Sci. Paris Sér. I Math. 306 (1988), 171-175.

[LP] M. L. Lapidus and C. Pomerance, Fonction zêta de Riemann et conjecture de Weyl-Berry pour les tambours fractals, C.R. Acad. Sci. Paris Sér. I Math. 310 (1990), 343-348.

[M11] R. B. Melrose, Weyl's conjecture for manifolds with concave boundary, Geometry of the Laplace Operator, Proc. Sympos. Pure Math., vol. 36, Amer. Math. Soc., Providence, R.I., 1980 , pp. 257-273.

[M12] _ The trace of the wave group, Contemp. Math., vol. 27, Amer. Math. Soc., Providence, R.I., 1984, pp. 127-167.

[Mt1] G. Métivier, Etude asymptotique des valeurs propres et de la fonction spectrale de problèmes aux limites, Thèse de Doctorat d'Etat, Mathématiques, Université de Nice, France, 1976.

[Mt2] _ Valeurs propres de problèmes aux limites elliptiques irréguliers, Bull. Soc. Math. France Mém. 51-52 (1977), 125-219.

[S1] Yu. G. Safarov, Asymptotics of the spectrum of a boundary value problem with periodic billiard trajectories, Funktsional Anal. i Prilozhen. 21 (1987), no. 4, 90-92; English transl. in Funct. Anal. Appl. 21 (1987).

[S2] _ Precise asymptotics of the spectrum of a boundary value problem and periodic billiards, Izv. Akad. Nauk SSSR Math. Ser. 52 (1988), no. 6, 1230-1251; English transl. in Math. USSR-Izv.

[V1] D. Vasil'ev, Asymptotics of the spectrum of a boundary value problem, Trudy Moskov. Mat. Obsch. 49 (1986), 167-237; English transl. in Trans. Moscow Math. Soc., 1987, pp. 173245.

[V2] _ One can hear the dimension of a connected fractal in $\mathbb{R}^{2}$, Petkov \& Lazarov, Integral Equations and Inverse Problems, Longman Academic, Scientific \& Technical, 1991, pp. 270-273. 
[W1] H. Weyl, Über die asymptotische Verteilung der Eigenwerte, Gött. Nach. (1911), 110-117.

[W2] _ Das asymptotische Verteilungsgesetz der Eigenwerte linearer partieller Differentialgleichungen, Math. Ann. 71 (1912), 441-479.

Universite Toulouse 1, place A. France, 31042 Toulouse Cedex, France

E-mail address: jfleck@earn.frcict81

Mathematics Subject Group, School of Mathematical and Physical Sciences, UniverSity OF SusSeX, Falmer, Brighton BN1 9QH, Great Britain

E-mail address: d.vassiliev@central.sussex.ac.uk 